\title{
Breast tuberculosis in outpatient practice: Case reports
}

\author{
Ndungu B.M., BSc, MBChB, MMed, Dip (MAS), Tharao M.K., BSc, Githaiga J.w., MBChB, MMed, \\ FCS (ECSA), Department of Human Anatomy, School of Medicine, University of Nairobi, Kenya and \\ Radia K., FR C Path, Pathcare Laboratory, Limuru road, Nairobi, Kenya \\ Corresponding author: Dr. Ndungu B.M., Department of Human Anatomy, School of Health Sciences, \\ University of Nairobi, P.O. Box 30197, Email: bmndungu@yahoo.com
}

\section{Summary}

There has been a general increase in both pulmonary and extra-pulmonary tuberculosis (TB) attributable to global spread of AIDS. The breast is rarely a focus whether primarily or contiguously. It is confused with cancer of the breast and confirmation by histology and/or demonstration of acid and alcohol fast bacilli (AAFB) is crucial. We present three cases of breast TB seen in a private surgical clinic within a two-year period. The diagnostic and treatment pathways are described and a review of literature on the disease presented.

\section{Introduction}

Breast TB is a rare condition first described by Sir Astley Cooper in 1829 (1). It is characterized by granulomatous inflammation of the breast parenchyma, which may present with cutaneous manifestations including skin changes and ulceration. The few published reports on the disease are from Asia where tuberculosis is still prevalent (2,3). In a 14-year study (1970-84) by Kedar et al (4), only 38 cases $(1.61 \%)$ were revealed out of 2230 resected breast specimens (4). In Western countries, the reported incidence is $0.1-0.25 \%$ (5). The factors thought to be responsible for the higher incidence of the disease in the developing world include increasing HIV/AIDS, an increase in the prevalence of multi-drug resistant tuberculosis and increasing age of lactating women (6).

Breast TB in Kenya has remained undocumented presumably due to under- recognition and where it is diagnosed, underreporting. Clinically and radiologically, breast TB can resemble a breast abscess, fibroadenoma or carcinoma (2). The most important of these differentials is carcinoma of the breast because of the inherent consequences of the treatment. The role of the laboratory in diagnosis of breast $\mathrm{TB}$ is therefore paramount. Histological confirmation requires demonstration of acid and alcohol fast bacilli (AAFB), culture or polymerase chain reaction (PCR) as means of identifying the causative mycobacterial organism.

This report illustrates evaluation of three cases of breast $\mathrm{TB}$ encountered in a private surgical/breast clinic and highlights the salient features that may aid in diagnosis.

\section{Case 1}

A 67 year old para $10+0$ presented with a one month history of a right breast lump. It was painless and slowly progressive in size. There were no skin changes, nipple distortion, discharge, axillary swelling and no history of a chronic illness. She had no significant breast cancer risk profile. Physical examination revealed a $3 \mathrm{~cm}$ diameter mass in the right upper outer quadrant. Her haemoglobin level was $14.5 \mathrm{~g} / \mathrm{dl}, \mathrm{WBC}$ count of $10.5 \times 10^{3} / \mu \mathrm{l}$ with a lymphocytosis of $42.9 \%$ and an ESR of $60 \mathrm{~mm} /$ hr. Mammography showed an irregular opacity in the right para-nipple region that appeared striated while the left breast was normal apart for some benign calcification (Figures 1 and 
2 respectively). Histology of a trucut biopsy showed inflammatory cells- lymphocytes and plasma cells. Microscopy with gram staining showed no organisms whereas culture and sensitivity yielded no growth. Due to the predominance of lymphocytes in the inflammatory cells, a Ziehl-Neelsen (ZN) stain was done on fresh tissue which turned out positive for AAFB (Figure 3). Two months after commencing anti-TB therapy, the lump had decreased in size to $1 \mathrm{~cm}$ diameter, the ESR was $50 \mathrm{~mm} / \mathrm{hr}$, WBC $10.2 \times 10^{3} / \mu \mathrm{l}$ with lymphocytes $36.5 \%$.

Figure 1: Right breast showing irregular opacity in the para-nipple region

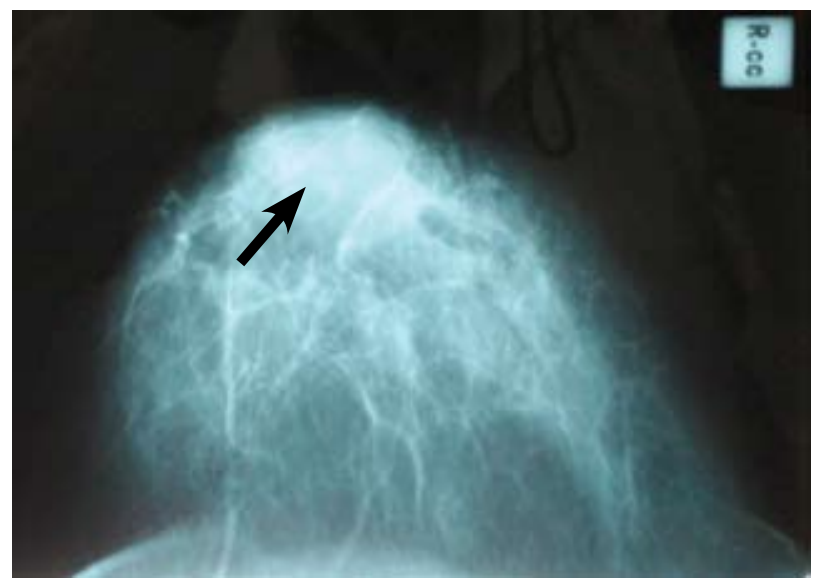

Figure 2: Left breast was normal. Some benign calcification present

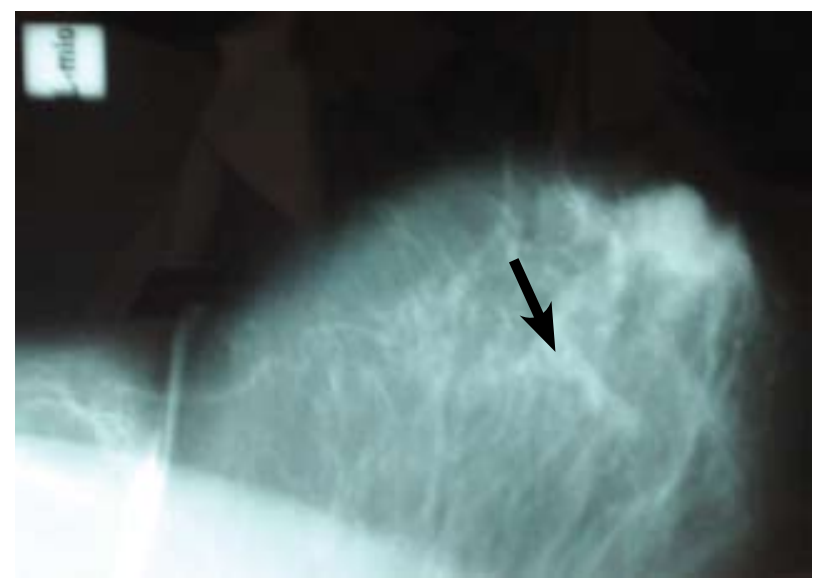

Figure 3: ZN stain of the patient showing the acid fast tubercle bacilli

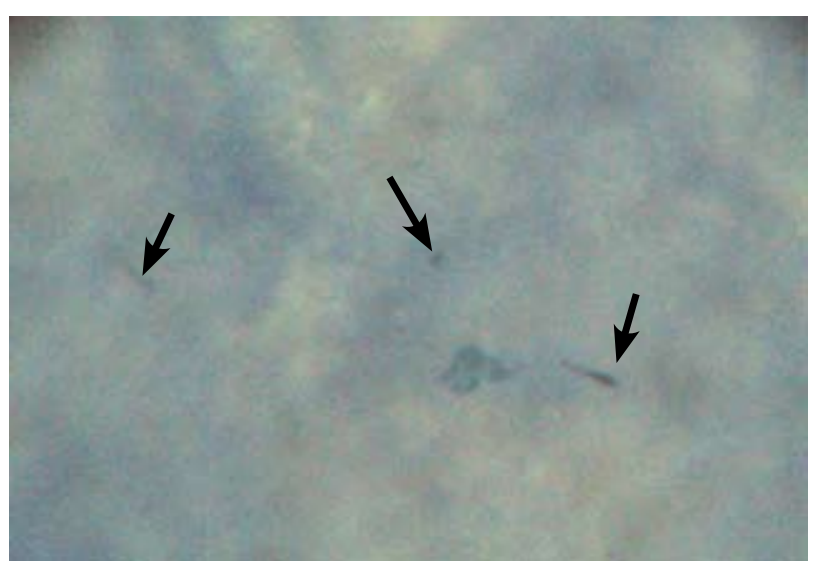

\section{Case 2}

A 35 year old para $2+1$ presented with one month history of a painful swelling on the left breast. It progressively increased in size and was associated with systemic symptoms of inflammation. There were no skin changes, no nipple distortion or discharge. Her breast cancer risk profile was insignificant and she regularly performed breast self examination. She had been on anti-TB drugs for pulmonary TB until two months prior to the visit.

Physical examination revealed an irregularly surfaced $3 \mathrm{~cm}$ diameter lump in the lower outer quadrant. Fine needle aspiration cytology (FNAC) was reported as TB mastitis. Core biopsy was advised but the patient left the country before this could be done due to personal reasons not related to the disease.

\section{Case 3}

A 26 year old para $1+0$ presented with a one month swelling on the right breast. It was painless, non- progressive and not associated with other local symptoms. Her last delivery was seven years earlier and had breastfed for two years. She had used medroxyprogesterone acetate and oral contraceptive pills for one year and did not perform breast self examination regularly. She was seroreactive for HIV markers but was not yet on antiretroviral drugs because of a CD4 $4^{+}$count of 450 cells $/ \mu 1$. 
Examination revealed a $3 \mathrm{~cm}$ lump in the upper outer quadrant with no axillary lymphadenopathy. A trucut biopsy revealed features consistent with TB (Figure 4) and she was started on anti-TB therapy. Subsequent follow up over a period of six months indicated reduction in the size of the lump. Haematological follow-up showed a decrease in ESR from $40 \mathrm{~mm} / \mathrm{hr}$ to $25 \mathrm{~mm} / \mathrm{hr}$ and leucocytosis from $14 \times 10^{3} / \mu \mathrm{l}$ to $9 \times 10^{3} / \mu \mathrm{l}$.

In all the three cases presented above, the main focus was from the breast rather than spread from surrounding ribs. A chest X-ray did not demonstrate any features of pulmonary TB.

Figure 4: Histologic section of a breast lump showing epithelioid cells $(\longrightarrow$ ) with caseous necrosis $(\mathrm{CN})$, (hematoxylin and eosin stained, original magnification $\times$ 200)

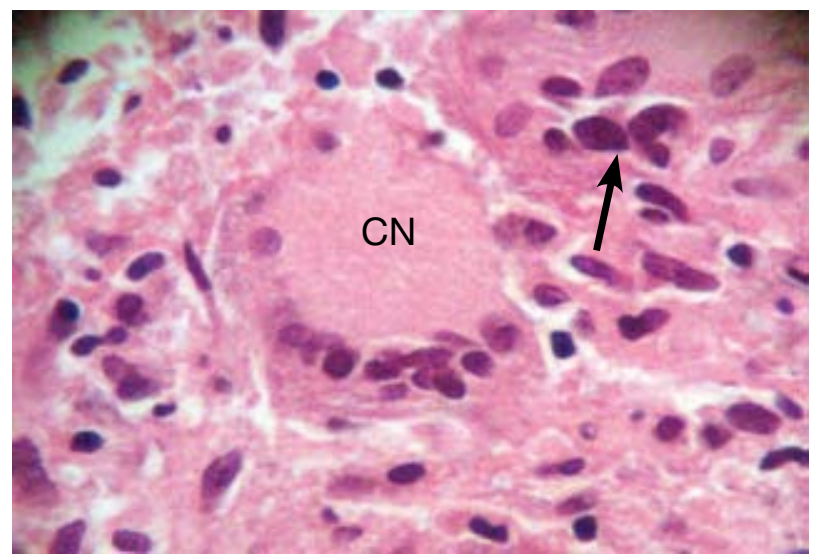

\section{Discussion}

Breast $\mathrm{TB}$ is a rare disease especially in the Western population. In the developing world however, the incidence is reported to be increasing due to increase in immunosuppresion principally due to HIV / AIDS and the emergence of multi-drug resistant TB. In our case study, only the third patient was seroreactive for HIV; however the CD4+ count was high enough to afford adequate immunity. The rarity of tuberculosis affecting the breast has been attributed to the mammary tissue offering resistance to the survival and multiplication of tubercular bacilli (7). A lump is the commonest presentation in breast $\mathrm{TB}(8)$. Constitutional symptoms in form of fever, weight loss, night sweats and failing general health and associated pulmonary tuberculous symptoms are uncommon (3). As in the patients in this report, presentation as multiple sinuses, ulcers and matted lymph nodes is uncommon and occurs late in the course of the disease (2).

Hamit et al (9) classified mammary TB into three mammographic patterns. The most common is nodular TB, which begins as a slowly growing indolent mass with or without lymphadenopathy. This caseating lesion resembles a fibroadenoma or carcinoma.

The diffuse type is characterized by multiple intercommunicating foci, and the mammographic picture may resemble that of inflammatory carcinoma.

The sclerosing type typically occurs in older women. Mammography reveals fibrotic dense breast tissue resembling a schirrous carcinoma $(9,10)$.

The 67 year-old patient presented in this report had mammographic features of the nodular type. Lesions due to TB have no specific ultrasonographic findings.

Definitive diagnosis of TB can only be made via demonstration of Mycobacterium organism through culture or PCR. However this either takes too long as in culture or can be too expensive for the patient as in the case of PCR. For these reasons diagnosis is often made through histological/cytological evidence of epithelioid granulomas, Langerhan giant cells and lymphohistiocytic aggregates $(3,13)$. Occasionally these features may be absent and the inflammatory nature of the histological specimen is non-specific. Kakker et al (11) could confidently diagnose breast tuberculosis in $73 \%$ of patients on the basis of FNAC findings of epithelioid cell granulomas with caseous necrosis. Cytological findings of granulomatous mastitis can also be found in plasma cell mastitis, fat necrosis, and actinomycosis (11). Other conditions that can give similar histological appearance include Wegener's granulomatosis, leprosy and syphilis.

Demonstration of the AAFB using the $\mathrm{ZN}$ stain usually confirms the diagnosis (12). However, in the presence of definitive histological/cytological changes, it is not 
mandatory for the Mycobacterium to be demonstrated microscopically using the $\mathrm{ZN}$ stain which only has a reliable sensitivity when the number of AAFB in the tissue is $10,000-$ $100,000 / \mathrm{ml}$ of material (8). If the histological features are characteristic of TB particularly the typical granulomas, then lack of demonstration of AAFB should not justify delay or denial of treatment. However if the patient presents with a breast abscess where the picture is dominated by acute inflammatory exudates, AFB positivity or histological confirmation is mandatory (8).

In the current case report, one case had a trucut histology which showed inflammatory cells, lymphocytes and plasma cells. Due to the predominance of lymphocytes in the inflammatory cells, a $\mathrm{ZN}$ stain was done which turned out positive for AAFB. Culture/ Sensitivity yielded no growth, which could have been due to the low numbers of bacilli in the sample material cultured or technical factors affecting yield of the culture. The second case had cytological features of TB mastitis whereas the third one had trucut features consistent with breast TB.

Currently literature advises that all breast lumps should be examined through FNAC, trucut biopsy or through excision biopsy. However when it comes to lumps suspected to be tuberculous in nature, the emphasis should be on FNAC and trucut biopsy to confirm the diagnosis. Excision is not required since the lump will often regress in size with antituberculous treatment (7). The yield of a trucut biopsy is superior to the FNAC and is relatively cost effective. Culture or PCR would provide definitive diagnosis. However as aforementioned, culture takes too long in our set-up and majority of our patients cannot be able to afford PCR.

The mainstay of treatment is the anti-TB drugs. Surgery is used in cases of drug resistance and/or involve extensive tissue damage (7). Wide excision of the granulomatous lesion or a simple mastectomy depending on the degree of disfigurement has proved to be effective (7).

All the three patients had no marked disfigurement or extensive tissue damage to warrant surgical intervention and the progress of the two patients still on follow-up on medical treatment is satisfactory.

\section{Conclusion}

The clinical presentation of breast $\mathrm{TB}$ is nonspecific and can be confused with breast carcinoma. FNAC and histology are suitable in diagnosing the disease. Treatment by anti-TB drugs should not be delayed as one waits for cultures. Confirmatory diagnosis using PCR is expensive for many patients.

\section{References}

1. Bedi U.S. and Bedi R.S. Bilateral breast tuberculosis. Indian J. Tuberculosis. 2001; 122: 215-217.

2. Sharma P.K., Babel A.L. and Yadav S.S. Tuberculosis of the breast (study of 7 cases). J. Postgrad. Med. 1991; 37: 24-26.

3. Khanna R., Prasanna G.V., Gupta P., et al. Mammary tuberculosis: Report on 52 cases. J. Postgrad. Med. 2002; 78: 422-424.

4. Kedar G.P., Bophate S.K. and Kherdekar M. Tuberculosis of the breast. Ind. J. Tub. 1985; 32: 146.

5. O'Reilly M., Patel K.R. and Cummins R. Tuberculosis of the breast presenting as carcinoma. Military Med. 2000; 165: 800-802.

6. Kenya demographic and Health Survey 2003; 59-60.

7. Bannerjee S.N., Ananthakrishran N., Mehta R.B., et al. Tuberculous mastitis: A continuing problem. World J. Surg. 1987; 11: 105-109.

8. Puneet, Satyendra K.T., Ritu R., Sanjay S., et al. Breast tuberculosis: Still common in India. J. Trop. Med. 2005; 2: 2.

9. Hamit H.F. and Ragsdale T.H. Mammary tuberculosis. (Editorial). J. R. Soc. Med. 1982; 75: 764-765.

10. Sabate J.M., Clotet M., Gomez A., et al. Radiologic evaluation of uncommon inflammatory and reactive breast disorders. Radiographics. 2005; 25: 411-424.

11. Kakker S., Kapila K., Singh M.K., et al. Tuberculosis of the breast. A cytomorphologic study. Acta. Cytol. 2000; 44: 292-296.

12. Benedito B.S., Lina G.D.S., Pedro V.L.C., et al. Clinical case report: Primary tuberculosis of the breast mimicking carcinoma. Amer. J. Trop. Med. Hyg. 2005; 73(5): 975-976. 